\title{
On the Continuity of Causal Automorphisms of Space-Time
}

\author{
J. L. Alonso and F. J. Yndurátn* \\ University of Zaragoza, Spain \\ Received October 1, 1966
}

\begin{abstract}
We prove that any causal automorphism of the (curved or not) spacetime in any dimension is continuous.
\end{abstract}

In a recent paper, ZeEMAN [1] has shown that relativistic invariance is implied by causality alone. More precisely, he has proved that the group of automorphisms of the Minkowski space $M_{4}$ preserving the partial ordering $x<y$ (we say that $x<y$ iff $(x-y) \cdot(x-y)>0$ and $x_{0}<y_{0}$ ) coincides with the group $G$ consisting of the connected Poincaré group plus space reversal and dilatations. Here $x=\left(x_{0}, x_{1}, x_{2}, x_{3}\right)$ is a vector in $M_{4}$ and $x \cdot y$ is the Lorentz scalar product of $x$ and $y$. Linearity or continuity are not assumed a priori.

ZEEMAN's proof leans essentially on the four-dimensional character of $M_{4}$, and, in fact (as pointed out by himself) the result fails for the $M_{2}$ space. On the other hand, the (pseudo) euclidean character of the whole of $M_{4}$ is also essential for his proof, and thus, as was to be expected, the theorem does not apply to the space-time of general relativity $\left(V_{4}\right)$ either. A less strong result, however, may still be shown to hold in the more general cases. In fact, we are going to prove:

Theorem. Let the $(n+1)$-dimensional Minkowski space with the metric $x \cdot y=\sum_{0}^{n} x_{\mu} y_{\nu} g_{\mu \nu}, g_{\mu \nu}=\operatorname{diag}(+1,-1, \ldots,-1)$ be denoted by $M_{n+1}$, and let the space $V_{n+1}$ be locally homeomorphic to $M_{n+1}$. If the (bijective) automorphism $f$ of $V_{n+1}$ is causal, then it is continuous.

We assume the euclidean topology for $M_{n+1}$. By locally homeomorphic we mean that for every $p$ in $V_{n+1}$ there exist a $x$ in $M_{n+1}$, two open neighborhoods of $p, x$, in $V_{n+1}, M_{n+1}$ denoted by $\Gamma, \Gamma^{\prime}$, and a mapping $\varphi: \Gamma \rightarrow \Gamma^{\prime}$ continuous and one-to-one such that $\varphi^{-1}$ is also continuous. Finally, to define causal mappings we proceed as follows: it is clear that, due to the topological manifold structure of $V_{n+1}$, we must define causality in terms of local causality. Thus we begin by defining locally causal mappings: $f$ is locally causal if a) for every point $p$ in $V_{n+1}$ and $x$ in $M_{n+1}$ there exists a $\Gamma$ around $p$ as above and the open

\footnotetext{
* Present address: Physics Department, New York University.
} 
set $\Lambda$ around $p$ such that both $\Lambda$ and $f \Lambda$ are contained in $\Gamma$ (locality); and b) given these $\Gamma, \Lambda$, and $\Gamma^{\prime}, \varphi$ being as above, the mapping $\varphi f \varphi^{-1}$ in $\varphi \Lambda$ is causal in the ordinary sense, i.e., it preserves the ordering $x<y\left(x<y\right.$ whenever $(x-y) \cdot(x-y)>0$ and $\left.x_{0}<y_{0}\right)$. Now, given a mapping $f$ of $V_{n+1}$, we say that it is causal if it may be decomposed as a product of locally causal mappings.

Remark 1. We may use the partial ordering just described or the ordering $x<\cdot y$ whenever $(x-y) \cdot(x-y)=0$ and $x_{0}<y_{0}$ (note that the last is not properly an ordering since it is not transitive), i.e., causality for massive or massless particles. The reason is that $f$ preserves one ordering iff it preserves the other (ZEEMAN's proof of this fact remains unaltered in the more general cases).

Remark 2. The requirement of $f$ being one-to-one is essential. It is not a difficult task to show this by explicit counterexamples.

We divide the proof of the Theorem into two steps:

1. Let $X$ be a topological space and let $\mathscr{B}$ be a basis of its topology (in the case of $M_{n+1}, \mathscr{B}$ may consist of the open balls). Let " $C$ " be a partial ordering in $X$; we write $x<y$ whenever $x<y$ but $y \llbracket x$. If $x<y$, we define the range $R_{x y}$ as the set of all $z$ with $x<z<y$. We say that the ordering is continuous in the case that the following be true: whenever $x<y$ there exists $B_{x}$ in $\mathscr{B}$ such that $x$ is in $B_{x}$ and $B_{x}<y$, and a $B_{y}$ also in $\mathscr{B}$ with $x<B_{y}$ and $y$ contained in $B_{y}$. One has:

Lemma. If (i) the ordering " $<$ " is continuous and (ii) for every $z$ in $B$ ( $B$ arbitrary in $\mathscr{B}$ ) there exist $x, y$ such that $R_{x y}$ is contained in $B$ and $z$ is in $R_{x y}$; if $f: X \rightarrow X$ is one-to-one and $f, f^{-1}$ preserve the ordering, then $f$ (and a fortiori, $f^{-1}$ ) is continuous.

Note 1. An alternative formulation of the Lemma would be the following: let $X$ be a topological space with an ordering " $<$ "; assume that a) for all $x, y$, the set $R_{x y}$ is open; and b) the sets $R_{x y}$ form a basis of the topology of $X$. Then, if $f$ is a one-to-one mapping of $X$, and if $f, f^{-1}$ preserve the ordering, they are continuous.

This form has the virtue of making apparent the simplicity of the Lemma; it is not difficult, on the other hand, to prove that both formulations are equivalent. For purposes of applications, however, we believe the former to be more appropriate.

Note 2 . The Lemma may be trivially generalised to mappings from a space $X_{1}$ to another $X_{2}$, both with topology and ordering.

The alternative formulation and generalisation of the Lemma are due to D. Ruelle.

Proof of the Lemma. Since for any $z$ in $R_{x y}$ there exist $B_{z}<y$ and $B_{z}^{\prime}>x, z$ being in both, the set $B=B_{z} \cap B_{z}^{\prime}$ is open, is contained in $R_{x y}$ and contains $z$. Consequently, $R_{x y}$ may be obtained as the union of such $B$ and thus it is open. It is also clear that, in virtue of the hypo- 
theses, for any open set $U$ and every point $z$ in $U$ there exists a $R_{x y}$ around $z$ and contained in $U$, i.e., any open set is the union of $R_{x y}$ 's. The continuity of $f, f^{-1}$ then follows from the fact that the transformed of a $R_{x y}$ by any of them is again a $R_{x^{\prime} y^{\prime}}$, and that a mapping is continuous whenever the inverse image of an open set is open.

2. To prove the Theorem it is now sufficient to show that the causality ordering verifies conditions (i), (ii) in the Lemma. As to the first, it is obvious in virtue of the continuity of the functions $x_{0}-y_{0}$ and $(x-y) \times$ $\times(x-y)$. Let us prove (ii); we will do it for $M_{n+1}$ as $X$. Let $z$ be in $B$ and take $x<z<y$ with $x, y$ lying on the parallel to the 0 -axis through $z$; let $d_{1}=y_{0}-z_{0}$ and $d_{2}=z_{0}-x_{0}$. Let finally $z^{\prime}$ be any point in $R_{x y}$. Now :

$$
\begin{aligned}
\left(d_{1}+d_{2}\right)^{2}= & \left(y_{0}-x_{0}\right)^{2}=\left(\left(y_{0}-z_{0}^{\prime}\right)+\left(z_{0}^{\prime}-x_{0}\right)\right)^{2}> \\
& >\left(y_{0}-z_{0}^{\prime}\right)^{2}+\left(z_{0}^{\prime}-x_{0}\right)^{2}>\sum_{1}^{n}\left(z_{i}^{\prime}-y_{i}^{\prime}\right)^{2}+\sum_{1}^{n}\left(x_{i}-z_{i}^{\prime}\right)^{2}> \\
& >\sum_{1}^{n}\left(z_{i}^{\prime}-z_{i}\right)^{2}>\max \left|z_{i}^{\prime}-z_{i}\right|^{2},
\end{aligned}
$$

the last because of $z$ being on the $x y$ line. On the other hand, $y_{0}-z_{0}^{\prime}>0$ and $z_{0}^{\prime}-x_{0}>0$, so that also $\left|z_{0}^{\prime}-z_{0}\right|<d_{1}+d_{2}$, i.e., $\left|z_{\mu}^{\prime}-z_{\mu}\right|<d_{1}+d_{2}$ for any $\mu$. It then suffices to take $d_{1}, d_{2}$ such that

to obtain that

$$
\left(d_{1}+d_{2}\right)^{2} \leqq \inf \left\{\sum_{0}^{n}\left|z_{\mu}-u_{\mu}\right|, u \notin B\right\} /(n+1)
$$

$$
\sum_{0}^{n}\left|z_{\mu}^{\prime}-z_{\mu}\right|<\inf \left\{\sum_{0}^{n}\left|z_{\mu}-u_{\mu}\right|, u \notin B\right\}
$$

i.e., that $R_{x y}$ is contained in $B$. This proves the Theorem for automorphisms of $M_{n+1}$. To prove it when $f$ is a locally causal automorphism of $V_{n+1}$, let $z$ be any point in $V_{n+1}$ and let $\Lambda, \Gamma, \Gamma^{\prime}$ and $\varphi$ be as before. Take $B$ contained into $\varphi \Lambda$; then, the previous analysis shows that $\varphi f \varphi^{-1}$ is continuous at $\varphi z$. The continuity of $f$ at $z$ is now clear since $f=\varphi^{-1}\left(\varphi f \varphi^{-1}\right) \varphi$, and all $\varphi, \varphi^{-1}$ and $\varphi f \varphi^{-1}$ are continuous, i.e., $f$ is the product of continuous mappings. Finally, in the most general case of any causal automorphism the theorem is proved by simply remarking that $f$ is a product of locally causal (and thus continuous) transformations.

We are grateful to Professors A. GALINDo and D. RuelLe for illuminating discussions; particularly to the last for pointing out an error of a former version of this paper as well as for the communication of the alternative version and generalisation of the Lemma.

\section{References}

[1] Zeeman, E. C.: J. Math. Phys. 5, 490 (1964).

25 Commun. math. Phys., Vol. 4 